\title{
Genomic and Transcriptomic Evidence for Carbohydrate Consumption among Microorganisms in a Cold Seep Brine Pool
}

\author{
Weipeng Zhang ${ }^{1 \dagger}$, Wei Ding ${ }^{1 \dagger}$, Bo Yang ${ }^{1}$, Renmao Tian ${ }^{1}$, Shuo Gu ${ }^{1}$, Haiwei Luo ${ }^{2}$ and \\ Pei-Yuan Qian ${ }^{1 *}$ \\ ${ }^{1}$ Division of Life Science, Hong Kong University of Science and Technology, Hong Kong, Hong Kong, ${ }^{2}$ Simon F. S. Li Marine \\ Science Laboratory, School of Life Sciences, Chinese University of Hong Kong, Shatin, Hong Kong
}

OPEN ACCESS

Edited by:

Chuanlun Zhang,

Tongji University, China

Reviewed by:

Tom O Delmont,

University of Chicago, USA

Meng Li,

Shenzhen University, China

*Correspondence:

Pei-Yuan Qian

boqianpy@ust.hk

${ }^{\dagger}$ These authors have contributed equally to this work.

Specialty section:

This article was submitted to

Aquatic Microbiology,

a section of the journal

Frontiers in Microbiology

Received: 10 September 2016

Accepted: 31 October 2016

Published: 15 November 2016

Citation:

Zhang W, Ding W, Yang B, Tian R,

Gu S, Luo H and Qian P-Y (2016)

Genomic and Transcriptomic Evidence

for Carbohydrate Consumption

among Microorganisms in a Cold

Seep Brine Pool.

Front. Microbiol. 7:1825.

doi: 10.3389/fmicb.2016.01825
The detailed lifestyle of microorganisms in deep-sea brine environments remains largely unexplored. Using a carefully calibrated genome binning approach, we reconstructed partial to nearly-complete genomes of 51 microorganisms in biofilms from the Thuwal cold seep brine pool of the Red Sea. The recovered metagenome-assembled genomes (MAGs) belong to six different phyla: Actinobacteria, Proteobacteria, Candidatus Cloacimonetes, Candidatus Marinimicrobia, Bathyarchaeota, and Thaumarchaeota. By comparison with close relatives of these microorganisms, we identified a number of unique genes associated with organic carbon metabolism and energy generation. These genes included various glycoside hydrolases, nitrate and sulfate reductases, putative bacterial microcompartment biosynthetic clusters (BMC), and $\mathrm{F}_{420} \mathrm{H}_{2}$ dehydrogenases. Phylogenetic analysis suggested that the acquisition of these genes probably occurred through horizontal gene transfer (HGT). Metatranscriptomics illustrated that glycoside hydrolases are among the most highly expressed genes. Our results suggest that the microbial inhabitants are well adapted to this brine environment, and anaerobic carbohydrate consumption mediated by glycoside hydrolases and electron transport systems (ETSs) is a dominant process performed by microorganisms from various phyla within this ecosystem.

Keywords: brine pool, biofilm, carbon metabolism, microbial genomics, transcriptomics

\section{INTRODUCTION}

Microorganisms play important roles in the biogeochemical cycles of deep-sea environments, such as deep-sea hydrothermal vents and brine pools. Studies of microbial lifestyles in brine pools are less common than those of hydrothermal vents. The brine pools studied so far include the Shaban Deep (Ferrer et al., 2012), Discovery Deep (Wang et al., 2013), Atlantis II Deep (Ngugi et al., 2015), and Kebrit Deep (Guan et al., 2015) of the Red Sea, which contains more than 25 deep hypersaline anoxic pools (Antunes et al., 2011). Previous investigations of other deep-sea brine pools included those in the Mediterranean Sea (van der Wielen et al., 2005) and the Gulf of Mexico (Formolo and Lyons, 2013). Brine pools probably share a common formation process of tectonically 
induced brine formation, resulting in frequently observed high methane and sulfite fluxes (Faber et al., 1998). As a consequence, the prevalent processes in the microbial inhabitants are anaerobic oxidation of methane and sulfite and sulfate reduction (Antunes et al., 2011). Surveys of brine pools identified a few widespread groups including Proteobacteria, Actinobacteria, Cyanobacteria, and Deferribacteres, and showed a higher diversity of bacteria over the archaea (Ferrer et al., 2012; Guan et al., 2015; Ngugi et al., 2015). Despite the common origin and shared features of the investigated brine pools, regional variation may lead to distinct combinations of physicochemical parameters and sustains microorganisms with unique adaptive strategies.

As important components of marine ecosystems, biofilms play an important role in ocean carbon transformation (Bhaskar and Bhosle, 2005; D'Arcy et al., 2013). However, biofilms are often very complex in nature. Heterogeneity of natural substrates induces heterogeneous biofilm development that is difficult to measure and observe (Claret, 1998). Devices for sampling deepsea biofilms are generally lacking, making their study challenging. To avoid these problems, one approach to studying the ecological roles of biofilms is to use artificial substrates to develop biofilms under steady-state conditions. This method has been used in previous studies of biofilm development in hydrothermal vent areas (Guezennec et al., 1998), estuary systems (Jones et al., 2007), and intertidal zones (Salta et al., 2013; Zhang et al., 2013).

Our previous investigation on the metagenomics of biofilms developed on artificial surfaces (Zhang et al., 2014, 2015) has illustrated the developmental processes of biofilms in a brine pool associated with the Thuwal cold seeps (Batang et al., 2012) on the floor of the Red Sea. This brine pool is located at a depth of approximately $850 \mathrm{~m}$ and is characterized by a high concentration of potential electron acceptors such as nitrate $\left(18.5 \mathrm{mg} \mathrm{L}^{-1}\right)$ and sulfate $\left(2.7 \mathrm{~g} \mathrm{~L}^{-1}\right)$. The particulate organic carbon (POC) concentration was $3.5 \mathrm{mg} \mathrm{L}^{-1}$ which was 10 times greater than that of the adjacent normal deepsea water (NDW) (Zhang et al., 2014, 2015). In contrast, methane and hydrogen sulfide were depleted with respect to NDW. Metagenomics were performed for biofilms developed on three different types of substrates: aluminum (Al), titanium (Ti) and polyvinyl chloride (PVC). Comparisons between brine and NDW biofilms indicated that polysaccharide metabolism related genes were highly enriched in the brine biofilms (Zhang et al., 2015). We also reconstructed two dominant bacterial genomes from the brine biofilm metagenomics datasets, a novel deltaproteobacterium and a novel epsilonproteobacterium. The former metagenome-assembled genome (MAG) possessed enhanced polysaccharide fermentation pathways, whereas the later possessed complete gene clusters for nitrate reduction and nitrogen fixation. We proposed that polysaccharide fermentation and proteolysis interacted with nitrogen cycling to form a complex chain for energy generation in these biofilms (Zhang et al., 2015).

Although, the biofilm development process in the Thuwal cold seep brine pool and the genomics of the two dominant microorganisms has been analyzed, the lifestyle of the other microorganisms in the brine biofilms remains unclear. In the present study, using the genome binning technique, we analyzed 51 partial to near-complete microbial genomes reconstructed from the $\mathrm{Al}$ and $\mathrm{PVC}$ biofilm metagenomes and the metatranscriptomes derived from these biofilms, toward a systematic understanding of microbial lifestyles in this ecosystem.

\section{MATERIALS AND METHODS Biofilm Sampling, DNA Extraction and Metagenomic Analysis}

Biofilm sampling was conducted in May 2013 in the Thuwal seeps II $\left(22^{\circ} 16 \mathrm{~N}-38^{\circ} 53 \mathrm{E}\right)$ by the remotely operated underwater vehicle (ROV) Max Rover, DSSI, USA during the Red Sea exploration cruise. Biofilms were developed on six different types of materials in the brine pool for 3 and 6 days. The devices for biofilm development has been reported in Zhang et al. (2014) and the schematic diagrams are also shown in Figure S1 in the present study. The materials were deployed into the brine pool by the ROV. Environmental parameters, such as temperature, salinity, and dissolved oxygen (DO) were measured using conductivitytemperature-depth (CTD) and SBE43 DO sensors attached to the ROV.

The biofilms used in the present study were developed on aluminum and PVC in the brine pool for 6 days and were referred to as "biofilm_Al and biofilm_PVC." Biofilms on these two materials were selected because biofilm_Al had the highest biomass and PVC represented a biofilm developed on plastic materials. Cells were recovered from the substrates using sterile cell scrapers and suspended in Tris- $\mathrm{HCl}$ buffer before extracting nucleic acid using the AllPrep DNA/RNA Mini Kit (Qiagen, Hilden, Germany). The extracted DNA was sequenced using an Illumina HiSeq 2000 platform. Metagenomic datasets of biofilm_Al and biofilm_PVC have been documented in Zhang et al. (2015), and the information of these two metagenomes are also listed in Table S2 in the present study. Assembly of the combined metagenomes of biofilm_Al and biofilm_PVC was performed using SPAdes Genome Assembler 3.6.1 (Bankevich et al., 2012). The specified $\mathrm{K}$ values for assembly included 21, $31,41,51,61,71$, and 81, and the "-careful" and "-pe" options were used. The metagenome-based taxonomic structures were obtained by ribosomal database project (RDP) classification (Wang et al., 2007) of the extracted 16S rRNA sequences, and the coverage information.

\section{Genome Binning and Validation}

Draft genome binning was conducted according to previously described methods (Albertsen et al., 2013; Tian et al., 2014). The genomes were separated mainly on the genome coverage, GC content, and tetranucleotide frequency. The Illumina reads were mapped to the contigs using Bowtie2 (version 2.0.0; Langmead and Salzberg, 2012). The genome coverage was calculated using SAMtools on a local server. The contigs were searched against a set of single-copy protein-encoding genes (Albertsen et al., 2013) using default cut-off values. The single-copy proteinencoding genes identified were searched against the National Center for Biotechnology Information non-redundant (NCBInr) database with BLASTP (e-value < 1e-07) with "xml" output 
format. Taxonomic information for the contigs was obtained by importing the BLAST results into MEGAN 5.0 (Huson et al., 2011). The metagenome pairs biofilm_Al and biofilm_PVC were selected to obtain the most effective separation and extraction of contigs. It must be noted that the diversity of the biofilm communities was not high and that many contigs belonging to different genomes formed clear, separate clusters based on coverage, facilitating genome binning and elimination of contamination (Figure S2).

Potential contig contamination was examined using CheckM (Parks et al., 2015), and PhylopythiaS, which is a sequence composition-based classifier that utilizes the hierarchical relationships between clades (Patil et al., 2012). Moreover, the Thaumarchaeota MAGs in the present study were compared with the eight genomes obtained using single-cell genomics by Ngugi et al. (2015), based on BLASTP (e-value $<1 \mathrm{e}-07$ ) and BLASTN (e-value $<1$ e-07) with predicted open reading frames (ORFs) as input. The results were visualized using the Artemis Comparison Tool (ACT; Carver et al., 2005).

\section{Genome Annotation}

The MAGs were annotated by searching the CarbohydrateActive EnZymes (CAZys) (Cantarel et al., 2009), Kyoto Encyclopedia of Genes and Genomes (KEGG) (Kanehisa and Goto, 2000), and Clusters of Orthologous Groups (COGs) (Galperin et al., 2014) databases. The ORFs in the MAGs were predicted using Prodigal (Hyatt et al., 2010) using a local server. Genes were annotated by BLASTP searches against the abovelisted databases using an e-value cut-off of 1e-07 on a local server. The online tool KAAS (KEGG Automatic Annotation Server) was also used to validate the KEGG annotation by local BLAST. Metabolic pathways were analyzed using online tools in KEGGMAPPER (http://www.genome.jp/kegg/mapper.html). Key enzymes revealed by KEGGMAPPER were rechecked against the NCBInr database based on the BLAST results.

\section{Phylogenetic Tree Construction}

In the phylogenetic analysis of the functional genes (e.g., polysaccharide deacetylase), the protein sequence alignment was determined by the molecular evolutionary genetics analysis using Muscle (MEGA 6.06; Tamura et al., 2013) with the following parameters: gap open -50 , cluster method UPGMB and min diagonal length 24. Gblock software (Castresana, 2000) was applied to eliminate less informative sites in the alignments. The construction of maximum-likelihood trees was conducted using MEGA 6.06 with the Tamura-Nei model, the Nearest-NeighborInterchange (NNI) method and 500 bootstrap replicates.

\section{RNA Extraction, cDNA Amplification and Metatranscriptomic Sequencing}

Biofilms on different substrates were harvested (10 slides per sample) onboard immediately after recovery using sterile cotton tips and stored in RNAlater buffer. RNA extraction was performed using the AllPrep DNA/RNA Mini Kit (Qiagen, Hilden, Germany). Bacterial cells in RNAlater buffer were pelleted by centrifugation at $4000 \mathrm{~g}$ for $10 \mathrm{~min}$ and then lysed with lysozyme, proteinase $\mathrm{K}$ and RLT buffer provided in the kit. Total nucleic acid was extracted, and DNA was removed using the DNA column provided with the AllPrep DNA/RNA Mini Kit. The integrity and quantity of the RNA were checked by agarose gel electrophoresis and a Nanodrop device (ND1000 spectrophotometer, DiaMed China Limited, Hong Kong), respectively. Prior to first-strand cDNA synthesis, genomic DNA contamination was further removed using the DNA-free ${ }^{\mathrm{TM}}$ DNA Removal Kit (Ambion, Austin, TX). The extracted RNA was then used as a template for whole-cDNA amplification using the Ovation Single-Cell RNA-Seq System, which enriches for non-rRNA during cDNA synthesis using proprietary whole transcriptome primers targeting non-rRNA sequences in the transcriptome. The synthesized cDNA was sequenced using an Illumina HiSeq 2000 platform.

\section{Metatranscriptomic Analysis}

The information for metatranscriptomic sequences are summarized in Table S2. The sequenced cDNA was subjected to quality control using the next-generation sequencing (NGS) QC toolkit (Patel and Jain, 2012). Assembly of the metatranscriptomic reads was performed using SPAdes Genome Assembler 3.6.1 (Bankevich et al., 2012) on a local server. The specified $K$ values $21,31,41,51,61,71$, and 81 were used under the "-careful" and "-pe" options. The ORFs were predicted following the steps mentioned above and annotated by BLASTP against the COG -database. To depict the gene expression profiling for individual microorganisms, the reads were aligned to contigs of the MAGs using Bowtie2 (version 2.0.0; Langmead and Salzberg, 2012). Gene expression abundance is the number of transcripts mapped to the CAZys of each MAG that normalized to the length of the genes.

\section{Data Accessibility}

The MAGs of Cloacimonetes were deposited in the Whole Genome Shotgun (WGS) database under accession numbers of SAMN04423165, SAMN04423166, SAMN04423167, and SAMN04423168. The MAGs of Marinimicrobia were deposited in the WGS database under accession numbers of SAMN04423157, SAMN04423158, SAMN04423159, SAMN04423160, SAMN04423161, SAMN04423162, and SAMN04423164. The MAG of Bathyarchaeota was deposited in the WGS database under accession number of SAMN04423169.

\section{RESULTS}

\section{Genome Information}

Genome binning using two metagenomes, biofilm_Al and biofilm_PVC, generated 51 MAGs of the biofilminhabited microorganisms (TCS1-51; "TCS" served as the abbreviation for "Thuwal cold seep"), including members of Actinobacteria (two MAGs belonging to Candidatus Microthrix), Gammaproteobacteria, Deltaproteobacteria, Epsilonprtoteobacteria, Candidatus Marinimicrobia, Candidatus Cloacimonetes, Bathyarchaeota (previously referred to as Miscellaneous Crenarchaeotic Group, MCG) and Thaumarchaeota (Table 1). Based on the metagenome-derived $16 \mathrm{~S}$ rRNA genes, these phyla were the dominant microbial 


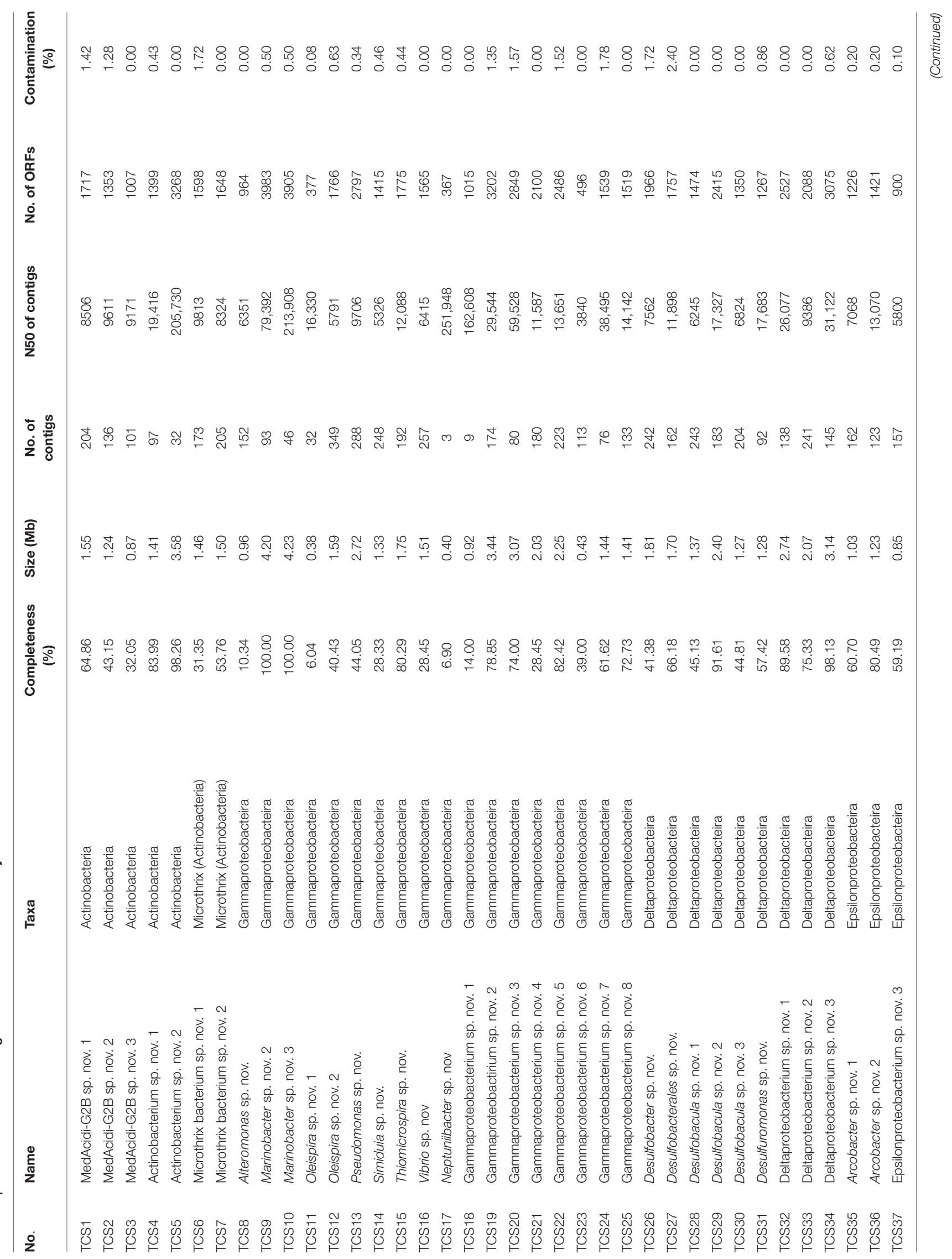




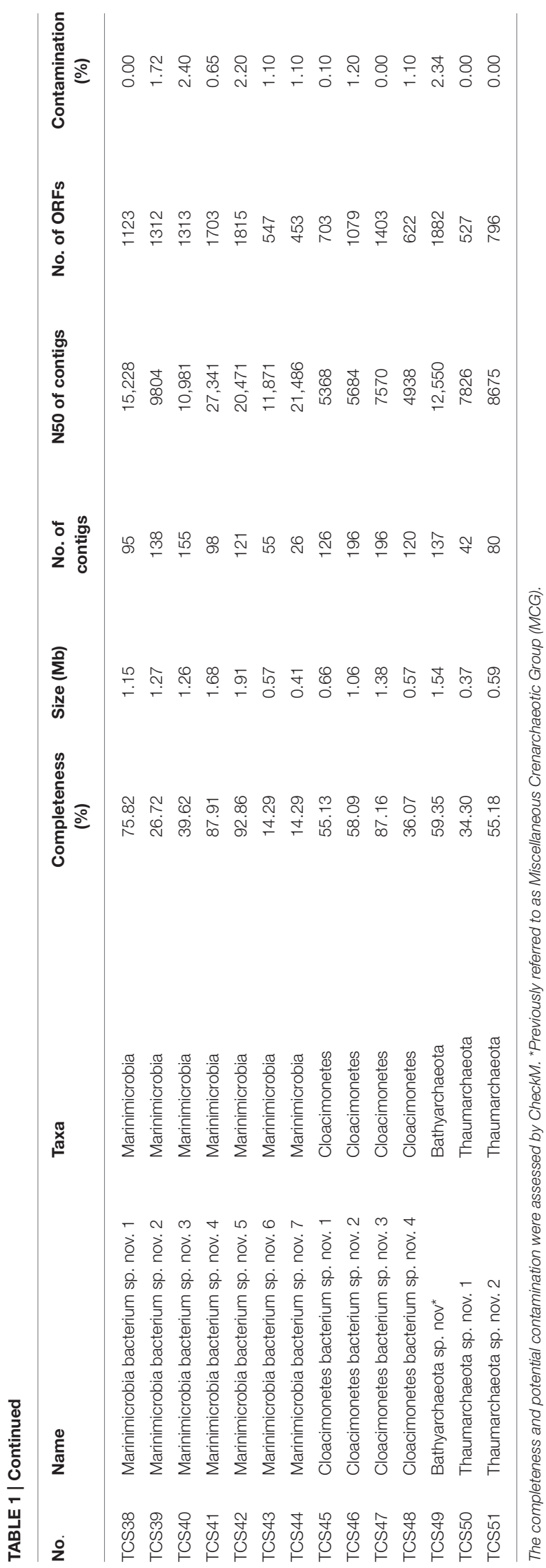

groups in the biofilms (Figure S3). According to the results of CheckM, these MAGs had a maximum completeness value of $100 \%$ and a maximum potential contamination of $2.4 \%$. Based on the results of PhylopythiaS, contigs of most of the MAGs were very consistent, as suggested by the percentage of contigs assigned to a defined taxonomy. For example, 97.2\% of the Pseudomonas sp. nov (TCS13) contigs could be assigned to the genus Pseudomonas. Moreover, contigs of the newly defined bacterial phyla, were compared with their relatives with complete or single-cell derived genomes to assess the potential contamination. For example, $95.8 \%$ of the Thaumarchaeota sp. nov. 1 (TCS50) and $91.3 \%$ of the Thaumarchaeota sp. nov. 2 (TCS51) could be assigned to the eight single-cell Thaumarchaeota genomes, which were from another brine pool in the Red Sea (Ngugi et al., 2015). The assigned ORFs were distributed in all of the contigs of the TCS MAGs as exemplified by Thaumarchaeota sp. nov. 1 (Figure S4). These results indicated that the MAGs reconstructed in the present study were of high quality.

\section{Central Metabolic Pathway}

A large proportion of the genes identified in many of the MAGs were found to be involved in fermentative carbohydrate metabolism. The distribution of CAZys (Cantarel et al., 2009) responsible for the degradation of complex carbohydrates is listed in Table S1, such as $\beta$-glucosidase (GH3), $\alpha$-amylase (GH13) and endo- $\beta$-1,4-galactanase (GH53). In total 17 unique CAZy categories corresponding to 1692 genes were identified in the 51 MAGs (on average 33 genes/genome). Genes encoding proteins playing a role in electron transport and energy production, including those encoding $\mathrm{F}_{420} \mathrm{H}_{2}$ dehydrogenase (fpo), cytochrome $\mathrm{C}$ (cytoC), the rhodobacter nitrogen fixation (rnf) complex, nitrate reductase and sulfate reductase, were identified in many of the MAGs. Annotation by COGs (Galperin et al., 2014) showed a high diversity in the gene arrangement of the $\mathrm{F}_{420}$ biosynthesis gene clusters (Figure S5), which were present in eight of the MAGs belonging to different phyla. Moreover, putative bacterial microcompartment (BMC) clusters, types of protein-bound bacterial organelles that function in carbon catabolism and storage (Nobu et al., 2016), were present in five MAGs including those of Actinobacteria, Marinimicrobia and Desulfobacter (Figure S6). As reported by Nobu et al. (2016), genes related to aldehyde and sugar metabolism are present in the BMC clusters, including rnf-C like NADH dehydrogenase (COG4656), sugar isomerase (COG0363) and aldehyde dehydrogenase (COG1012). However, other genes were also identified in the present study, such as NTP pyrophosphohydrolases and oxidative damage repair enzymes (COG0494), making the BMC clusters different from those in previous study.

The prevalence and versatility of carbohydrate metabolism and energy generation was further supported by KEGG-based pathway reconstruction. The central metabolic strategies are summarized in Figure 1. Many of the MAGs encoded proteins associated with monosaccharide and polysaccharide transport, nitrate and sulfate reduction, electron transport systems (ETSs), glycolysis, and reoxidation of energy-conserving elements via 

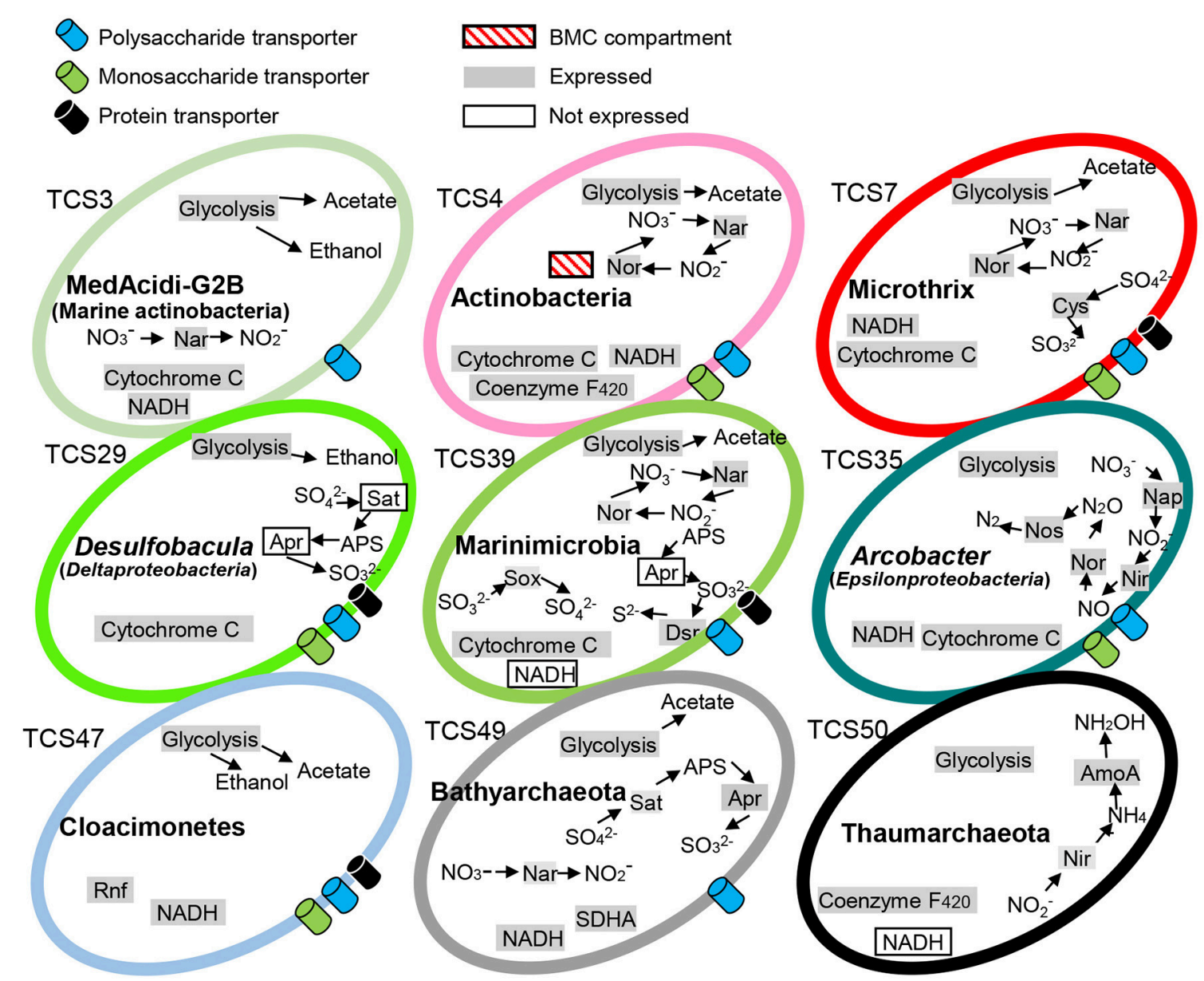

FIGURE 1 | Metabolic capacities of representative microorganisms based on genomic and metatranscriptomic analyses. The complete pathways responsible for carbohydrate fermentative metabolism as well as energy generation were detected in the MAGs. The expression profiles of these pathways were examined by mapping transcripts to key functional genes. TCS, Thuwal cold seep; Rnf, Rhodobacter nitrogen fixation; BMC, putative bacterial microcompartment; SDHA, succinate dehydrogenase complex subunit A. The 51 MAGs were numbered TCS1-51.

acetyl-CoA reduction to ethanol or acetate. For example, monosaccharide and polysaccharide transport was indicated by the presence of a number of $\mathrm{ABC}$ transporters in all the bacterial genomes; dissimilatory nitrate reduction was indicated by both respiratory nitrate reductase (nar) and periplasmic nitrate reductases (nap); dissimilatory sulfate reduction was indicated by sulfur oxidation (SOX) gene cluster and adenylylsulfate reductase; and acetate generation was indicated by acetate kinase. Notably, the Marinimicrobia bacterium sp. nov. 2 (TCS39) and Bathyarchaeota sp. nov (TCS49) possessed both dissimilatory nitrogen and sulfur reduction pathways.

\section{Unique Genomic Features}

When the TCS MAGs were compared with reference genomes within the same phylum, unique features concerning carbohydrate metabolism and energy generation were observed. For example, genes encoding NADH dehydrogenase, such as nuoB, nuoL, ndufv2, NdhJ, and NoxF were only identified in four Cloacimonetes MAGs (TCS45-47) when compared with complete or single-cell Cloacimonetes genomes (Figure S7); similar results were observed for chitin deacetylase (EC3.5.1.41) and the $\alpha$-D-glucose transformation pathway, which are important steps in glycolysis. Moreover, the complete pyruvate to acetate pathway was identified in Marinimicrobia bacterium sp. nov. 2 (TCS39) and Marinimicrobia bacterium sp. nov. 3 (TCS40), but the enzymes included in this pathway could not be found in the four reference genomes, the singlecell Marinimicrobia genomes from previous studies (Rinke et al., 2013; Nobu et al., 2015) (Figure S8). In addition, the Bathyarchaeota MAG (TCS49) harbored specific genes for chitin, cellulose, and $\beta$-D-glucose metabolism, such as a glycoside hydrolase family 3 gene (probably chitin deacetylase; EC3.5.1.41) and $\beta$-glucosidase (EC3.2.1.4) (Figure S9); the presence of aprAB and $c y s C$ genes, which are involved in dissimilatory sulfate reduction, differentiated TCS49 from the reference genomes.

Given that a number of unique genes were identified from these MAGs as compared with their relatives, we examined the phylogeny of these genes. The polysaccharide deacetylase 
(EC3.2.1.21) in Cloacimonetes bacterium sp. nov. 1 (TCS45) was phylogenetically close to the polysaccharide deacetylase from Lactobacillus ceti (Figure 2). Although, the bootstrap values in the tree were not high, BLAST analysis also revealed close relationship between polysaccharide deacetylase in Cloacimonetes bacterium sp. nov. 1 and those from firmicutes. The nitrate reductase $\alpha$ and $\beta$ units in Marinimicrobia bacterium sp. nov. 3 (TCS40) were phylogenetically close to the nitrate reductases from Geothrix fermentans (Figures S10, S11). In addition, the glycoside hydrolase family 3 gene in Bathyarchaeota sp. nov (TCS49) was affiliated with Hungatella hathewayi and
Clostridium hathewayi (Figure S12). The adjacent genes in the same contigs, which were affiliated with Cloacimonetes, Marinimicrobia or Bathyarchaeota, were displayed to confirm that the presence of these novel genes was probably not attributable to contig contamination.

\section{Genome-Wide Expression Profiling}

To explore the major active functions, we obtained $\sim 600$ $\mathrm{Mb}$ of metatranscriptomic datasets for the biofilms developed on $\mathrm{Al}$ and PVC. The metatranscriptomic sequences were searched against the COG database, revealing an overall
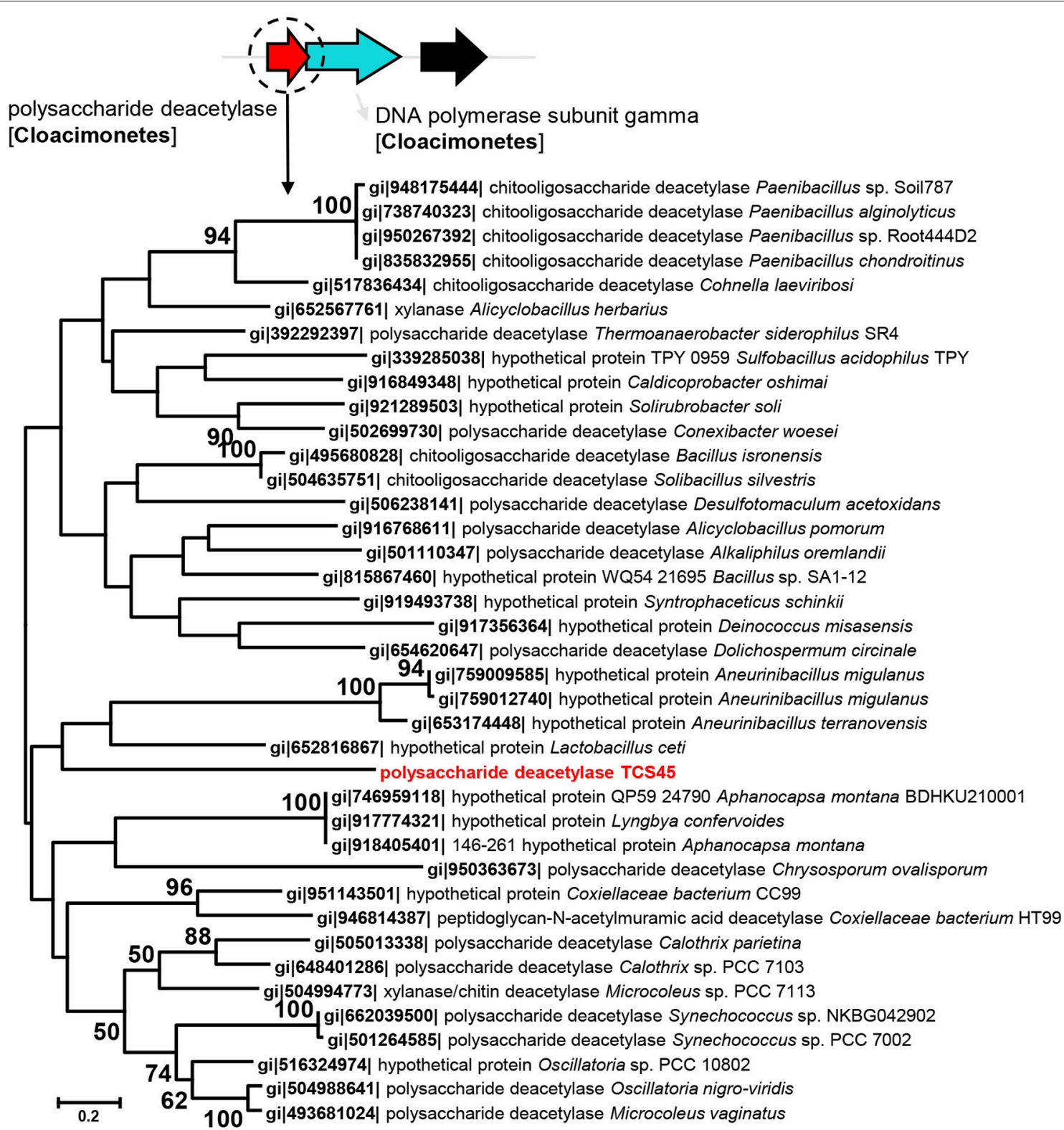

FIGURE 2 | Maximum likelihood phylogenetic tree of the polysaccharide deacetylase in Cloacimonetes bacterium sp. nov. 1. The reference sequences were obtained from NCBI databases. Bootstrap values based on 500 replicates are shown at the nodes. The taxonomic affiliation of the adjacent genes, based on the best hits in the BLASTP search against the NCBInr database are also shown to eliminate the possibility of contig contamination. 
picture of the active functions. Transcripts of the genes involved in carbohydrate transport and metabolism were among the most abundant in the metatranscriptomes, particularly the glycosidases (COG0366), $\beta$-glucosidase-related glycosidases (COG1472) and $\beta$-galactosidase/beta-glucuronidase (COG3250) (Figure 3). In contrast, few peptidases that are essential for protein metabolism were identified, and rank analysis suggested relative lower expression levels for these genes (Figure S13).

The reads from these two metatranscriptomes were mapped to the 51 MAGs. Abundant active bacteria could be identified for nearly all of the phyla, whereas rare active bacteria were also included in each phylum. Key functional genes involved in nitrate and sulfate respiration were expressed, such as the napAB, narGH, nirK, norC, nosZ, and sulfur oxidation (SOX) gene cluster. The gene expression information was integrated in to pathways shown in Figure 1. Moreover, when the metatranscriptomic sequences were mapped to CAZys in each MAG, a heatmap showing expression pattern of CAZys was generated (Figure 4). The results confirmed the expression of diverse carbohydrate metabolism related genes, and indicated relative higher expression of the glycoside hydrolases (GHs), glycosyltransferase (GTs) than enzymes in other families.

\section{DISCUSSION}

We reconstructed 51 partial to nearly complete MAGs with the aim of investigating the microbial lifestyles in brine pool biofilm communities. We validated the genome binning results using different approaches before the subsequent analyses. Genomic and transcriptomic analyses provided evidence that organic carbon metabolism utilizing nitrate, nitrite, and sulfate as potential electron acceptors is prevalent among these microorganisms. In particular, we identified several unique metabolic pathways for Cloacimonetes, Marinimicrobia, and Bathyarchaeota, which differ from previously reported lifestyles for these microbial groups.

It is important to validate the results from genome binning, although this approach has been repeatedly employed in various studies (Brown et al., 2015; Emerson et al., 2016). We suggest that validation using PhylopythiaS is suitable for genome bins of well-documented microbial taxa, such as Pseudomonas. But for novel phyla without many available genomes in public databases, comparing to single-cell amplified genomes (SAGs) from similar habitats is a more effective approach. The Thaumarchaeota genome binned in the present study possesses a gene inventory that is nearly identical to the SAGs from another Red Sea brine pool (Ngugi et al., 2015); this is strong evidence that the genome binning approach employed here is highly accurate.

The overall profiling of the genomics suggested the prevalence of organic carbon metabolism using diverse electron transfer elements. This notion is supported by the high diversity or specificity of particular pathways. Previous studies have discussed the distribution of $\mathrm{F}_{420}$ biosynthesis genes in the genomes of microorganisms other than Archaea and Actinobacteria (Selengut and Haft, 2010; Li et al., 2014). In the present study, we showed a higher diversity of the $\mathrm{F}_{420}$ biosynthesis
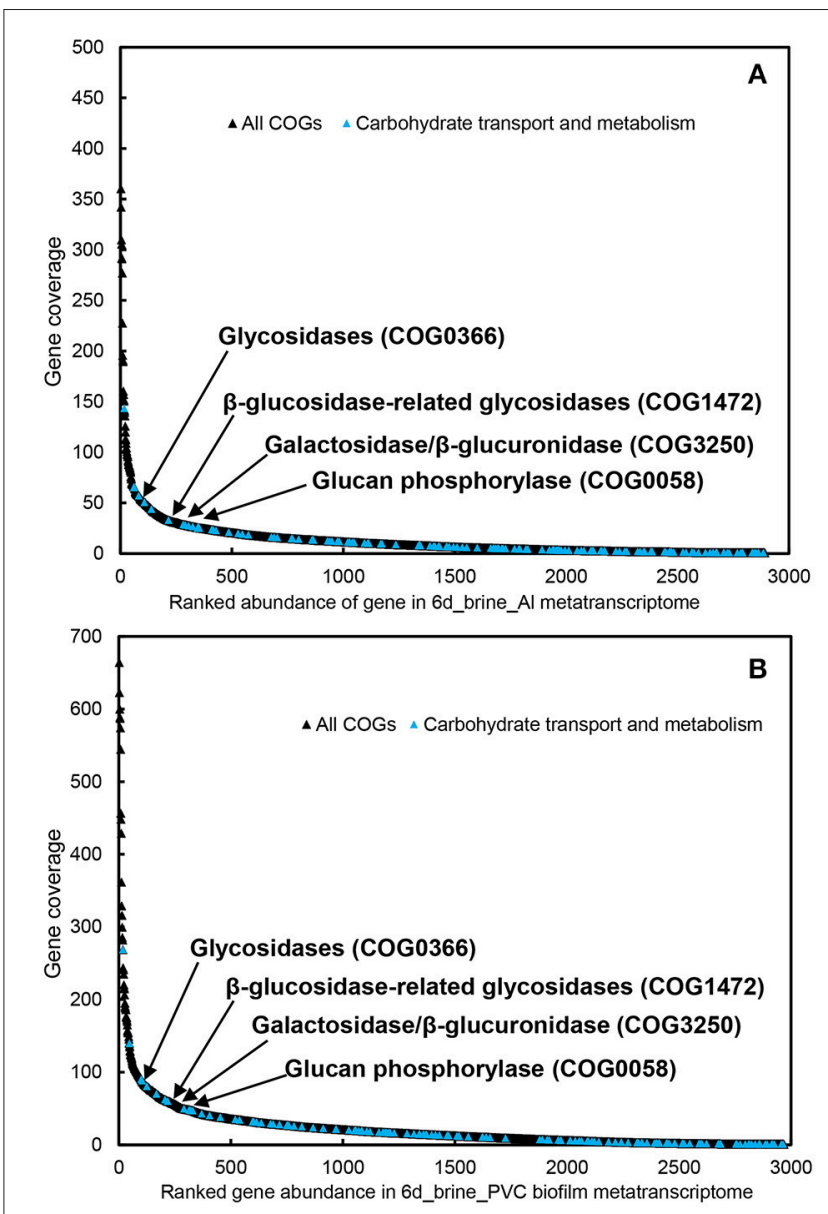

FIGURE 3 | Transcript abundance of all COGs and carbohydrate transport and metabolism genes in the two biofilm

metatranscriptomes (A, biofilm_Al; B, biofilm_PVC). Genes for Glycosidases (COG0366), $\beta$-glucosidase-related glycosidases (COG1472),

galactosidase/ $\beta$-glucuronidase (COG3250), and glucan phosphorylase (COG0058) are highlighted in the figure.

gene cluster than previously assumed, suggesting a possible correlation between these genes and microbial adaptation to the brine pool. The presence of BMC compartments provides additional evidence that carbohydrate metabolism is particularly important for microbial diversity in this habitat, consistent with the concept that carbon storage is a mechanism conferring microbial resistance to hypersaline stresses (Werner et al., 2014). Notably, environmental adaptation of the Thuwal cold seep brine residents seems to have been facilitated by horizontal gene transfer (HGT) from various bacteria, as indicated by the phylogeny of several novel genes in the genome bins. The metatranscriptomic results suggested that anaerobic carbohydrate catabolism modulates not only genomic content but also post-genomic activity.

With respect to the detailed microbial lifestyles, this study is the first to report the presence of certain fermentative groups in a brine environment. To date, Microthrix have only been recorded in activated sludge ecosystems (Muller et al., 


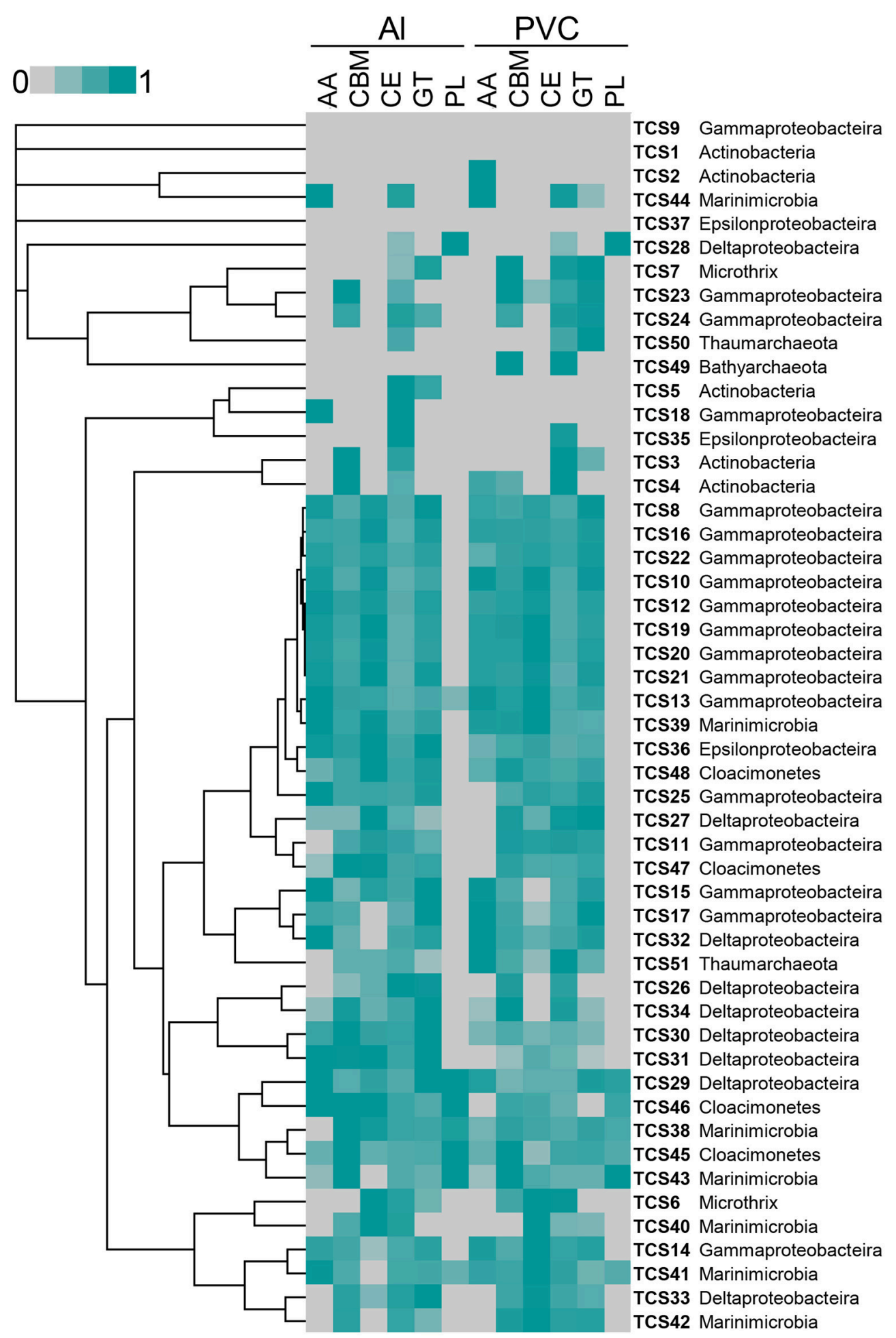

FIGURE 4 | Relative abundance of expressed genes for carbohydrate metabolism from each MAG. Expression levels for auxiliary activities (AAs), carbohydrate-binding module (CBMs), carbohydrate esterases (CEs), glycoside hydrolases (GHs), Glycosyltransferase (GTs), and polysaccharide lyases (PLs) from each MAG in the biofilm_Al and biofilm_PVC metatranscriptomes are shown. Gene expression abundance is the number of transcripts mapped to the CAZys of each MAG that normalized to the length of the genes. The highest expression level was normalized as "1." 
2012; McIlroy et al., 2013); Actinobacterium MedAcidi-G2B, for which little genomic information is available, have been reported only in the deep-sea habitat of the Mediterranean Sea (Mizuno et al., 2015); and Cloacimonetes are usually found in wastewater treatment plants or bioreactors (Pelletier et al., 2008; Nobu et al., 2015). Cloacimonetes and Marinimicrobia were found to be proteolytic amino-acid degraders and syntrophic propionate degraders, respectively (Nobu et al., 2015). However, the genomic and metatranscriptomic analyses of the present study showed their capacity to perform carbohydrate fermentation. The Bathyarchaeota may also be able to degrade polysaccharides, as suggested by the presence and expression of unique chitinase, cellulase and a rather complete acetate fermentation pathway in the MAG. The Bathyarchaeota MAG harbors a dissimilatory sulfate reduction pathway, differentiating it from other Bathyarchaeota members (Lloyd et al., 2013; Meng et al., 2014; Fillol et al., 2016) and suggesting the metabolic plasticity of this archaeal group. Nitrate or sulfate reducers, such as unclassified members of the Deltaproteobacteria and Epsilonproteobacteria, are also probable important players in polysaccharide fermentation, supporting the metabolic interconnection of carbohydrate metabolism with sulfur and nitrogen cycles in the brine pool.

\section{CONCLUSION}

Microbial consumption of organic carbons that sink from the surface ocean to deep-sea environments contributes to the remineralization of so called "dead organic matter" or "fecal material." Although, organic carbon consumption by deep-sea bacteria and archaea has not been quantified, our findings demonstrated the participation of diverse and ubiquitous

\section{REFERENCES}

Albertsen, M., Hugenholtz, P., Skarshewsk, A., Nielsen, K. L., and Tyson, G. W. (2013). Genome sequences of rare,uncultured bacteria obtained by differential coverage binning of multiple metagenomes. Nat. Biotechnol. 31, 533-538. doi: $10.1038 /$ nbt. 2579

Antunes, A., Ngugi, D. K., and Stingl, U. (2011). Microbiology of the Red Sea (and other) deep-sea anoxic brine lakes. Environ. Microbiol. Rep. 3, 416-433. doi: 10.1111/j.1758-2229.2011.00264.x

Bankevich, A., Nurk, S., Antipov, D., Gurevich, A. A., Dvorkin, M., Kulikov, A. S., et al. (2012). SPAdes: a new genome assembly algorithm and its applications to single-cell sequencing. J. Comput. Biol. 19, 455-477. doi: $10.1089 / \mathrm{cmb} .2012 .0021$

Batang, Z. B., Papathanassioub, E., Al-Suwailema, A., Smithb, C., Salomidib, M., Petihakisb, G. M. et al. (2012). First discovery of a cold seep on the continental margin of the central Red Sea. J. Mar. Syst. 94, 247-253. doi: 10.1016/j.jmarsys.2011.12.004

Bhaskar, P. V., and Bhosle, N. B. (2005). Microbial extracellular polymeric substances in marine biogeochemical processes. Curr. Sci. 88, 45-53.

Brown, C. T., Hug, L. A., Thomas, B. C., Sharon, I., Castelle, C. J., Singh, A., et al. (2015). Unusual biology across a group comprising more than $15 \%$ of domain Bacteria. Nature 523, 208-211. doi: 10.1038/nature14486

Cantarel, B. L., Coutinho, P. M., Rancurel, C., Bernard, T., Lombard, V., and Henrissat, B. (2009). The Carbohydrate-Active EnZymes database (CAZy): microbial taxa in this process. The niche-specific functions suggest a long history of microbial adaptation to the brine pool, where anaerobic organic carbon metabolism is likely to be an important driving force. Our findings shed new light on the roles of microorganisms in carbon cycling in deep-sea environments.

\section{AUTHOR CONTRIBUTIONS}

PQ: Designed the study; WZ, WD, BY, RT, SG, and HL: Performed the analyses. All authors wrote the manuscript and the Supplementary Materials.

\section{ACKNOWLEDGMENTS}

The authors are grateful to Dr. Abdulaziz M Al-Suwailem and his team in the Marine Core Laboratory of the King Abdulaziz University of Science and Technology for their technical support in the field cruise and constructing experimental apparatus and the crew of R/V Aegaeo for providing technical assistance during sample collection. This study was supported by awards from the Sanya Institute of Deep Sea Science and Engineering, the Chinese Academy of Sciences (SIDSSE, CAS) (SIDSSE201206), the National Basic Research Program of China (973 Program, No. 2012CB417304), the GRC grant (661611) of HKSAR government, and the King Abdullah University of Science and Technology (SA-C0040/UK-C0016) to PQ.

\section{SUPPLEMENTARY MATERIAL}

The Supplementary Material for this article can be found online at: http://journal.frontiersin.org/article/10.3389/fmicb. 2016.01825/full\#supplementary-material an expert resource for glycogenomics. Nucleic Acids Res. 37, 233-238. doi: 10.1093/nar/gkn663

Carver, T. J., Rutherford, K. M., Berriman, M., Rajandream, M. A., Barrell, B. G., and Parkhill, J. (2005). ACT: the Artemis comparison tool. Bioinformatics 21, 3422-3423. doi: 10.1093/bioinformatics/bti553

Castresana, J. (2000). Selection of conserved blocks from multiple alignments for their use in phylogenetic analysis. Mol. Biol. Evol. 17, 540-552. doi: 10.1093/oxfordjournals.molbev.a026334

Claret, C. (1998). A method based on artificial substrates to monitor hyporheic biofilm development. Int. Rev. Hydrobiol. 83, 135-143. doi: 10.1002/iroh.19980830204

D'Arcy, R., Amend, J. P., and Osburn, M. R. (2013). Microbial diversity and potential for arsenic and iron biogeochemical cycling at an arsenic rich, shallow-sea hydrothermal vent (Tutum Bay, Papua New Guinea). Chem. Geol. 348, 37-47. doi: 10.1016/j.chemgeo.2012.02.024

Emerson, J. B., Thomas, B. C., Alvarez, W., and Banfield, J. F. (2016). Metagenomic analysis of a high carbon dioxide subsurface microbial community populated by chemolithoautotrophs and bacteria and archaea from candidate phyla. Environ. Microbiol. 18, 1686-1703. doi: 10.1111/1462-2920. 12817

Faber, E., Botz, R., Poggenburg, J., Schmidt, M., Stoffers, P., and Hartmann, M. (1998). Methane in Red Sea brines. Org. Geochem. 29, 363-379. doi: 10.1016/S0146-6380(98)00155-7

Ferrer, M., Werner, J., Chernikova, T. N., Bargiela, R., Fernández, L., La Cono, V., et al. (2012). Unveiling microbial life in the new deep-sea hypersaline Lake 
Thetis. Part II: a metagenomic study. Environ. Microbiol. 14, 268-281. doi: 10.1111/j.1462-2920.2011.02634.x

Fillol, M., Auguet, J. C., Casamayor, E. O., and Borrego, C. M. (2016). Insights in the ecology and evolutionary history of the Miscellaneous Crenarchaeotic Group lineage. ISME J. 10, 665-677. doi: 10.1038/ismej.2015.143

Formolo, M. J., and Lyons, T. W. (2013). Sulfur biogeochemistry of cold seeps in the Green Canyon region of the Gulf of Mexico. Geochim. Cosmochim. Acta 119, 264-285. doi: 10.1016/j.gca.2013.05.017

Galperin, M. Y., Makarova, K. S., Wolf, Y. I., and Koonin, E. V. (2014). Expanded microbial genome coverage and improved protein family annotation in the COG database. Nucleic Acids Res. 43, D261-D269. doi: 10.1093/nar/gku1223

Guan, Y., Hikmawan, T., Antunes, A., Ngugi, D., and Stingl, U., (2015). Diversity of methanogens and sulfate-reducing bacteria in the interfaces of five deep-sea anoxic brines of the Red Sea. Res. Microbiol. 166, 688-699. doi: 10.1016/j.resmic.2015.07.002

Guezennec, J., Ortega-Morales, O., Raguenes, G., and Geese, G. (1998). Bacterial colonization of artificial substrate in the vicinity of deep-sea hydrothermal vents. FEMS Microbiol. Ecol. 26, 89-99. doi: 10.1111/j.15746941.1998.tb00495.x

Huson, D. H., Mitra, S., Ruscheweyh, H. J., Weber, N., and Schuster, S. C. (2011). Integrative analysis of environmental sequences using MEGAN4. Genome Res. 21, 1552-1560. doi: 10.1101/gr.120618.111

Hyatt, D., Chen, G. L., LoCascio, P. F., Land, M. L., Larimer, F. W., Hauser, L. J., et al. (2010). Prodigal: prokaryotic gene recognition and translation initiation site identification. BMC Bioinformatics 11:119. doi: 10.1186/1471-2105-11-119

Jones, P. R., Cottrell, M. T., Kirchman, D. L., and Dexter, S. C. (2007). Bacterial community structure of biofilms on artificial surfaces in an estuary. Microb. Ecol. 53, 153-162. doi: 10.1007/s00248-006-9154-5

Kanehisa, M., and Goto, S. (2000). KEGG: kyoto encyclopedia of genes and genomes. Nucleic Acids Res. 28, 27-30. doi: 10.1093/nar/28.1.27

Langmead, B., and Salzberg, S. L. (2012). Fast gapped-read alignment with Bowtie 2. Nat. Methods 9, 357-359. doi: 10.1038/nmeth.1923

Li, X., Feng, F., and Zeng, Y. (2014). Genome of betaproteobacterium Caenimonas sp. strain SL110 contains a coenzyme F420 biosynthesis gene cluster. J. Microbiol. Biotechnol. 24, 1490-1494. doi: 10.4014/jmb.1405.05039

Lloyd, K. G., Schreiber, L., Petersen, D. G., Kjeldsen, K. U., Lever, M. A., Steen, A. D., et al. (2013). Predominant archaea in marine sediments degrade detrital proteins. Nature 496, 215-218. doi: 10.1038/nature12033

McIlroy, S. J., Kristiansen, R., Albertsen, M., Karst, S. M., Rossetti, S., Nielsen, J. L., et al. (2013). Metabolic model for the filamentous 'Candidatus Microthrix parvicella' based on genomic and metagenomic analyses. ISME J. 7, 1161-1172. doi: 10.1038/ismej.2013.6

Meng, J., Xu, J., Qin, D., He, Y., Xiao, X., and Wang, F. (2014). Genetic and functional properties of uncultivated MCG archaea assessed by metagenome and gene expression analyses. ISME J. 8, 650-659. doi: 10.1038/ismej.2013.174

Mizuno, C. M., Rodriguez-Valera, F., and Ghai, R., (2015). Genomes of planktonic Acidimicrobiales: widening horizons for marine Actinobacteria by Metagenomics. mBio 6, e02083-e02014. doi: 10.1128/mBio.02083-14

Muller, E. E., Pinel, N., Gillece, J. D., Schupp, J. M., Price, L. B., Engelthaler, D. M., et al. (2012). Genome sequence of 'Candidatus Microthrix parvicella' Bio17-1, a long-chain-fatty-acid-accumulating filamentous actinobacterium from a biological wastewater treatment plant. J. Bacteriol. 194, 6670-6671. doi: 10.1128/JB.01765-12

Ngugi, D. K., Blom, J., Alam, I., Rashid, M., Ba-Alawi, W., Zhang, G., et al. (2015). Comparative genomics reveals adaptations of a halotolerant thaumarchaeon in the interfaces of brine pools in the Red Sea. ISME J. 9, 396-411. doi: 10.1038/ismej.2014.137

Nobu, M. K., Dodsworth, J. A., Murugapiran, S. K., Rinke, C.,Gies, E. A., Webster, G., et al. (2016). Phylogeny and physiology of candidate phylum 'Atribacteria' (OP9/JS1) inferred from cultivation-independent genomics. ISME J. 10, 273-286. doi: 10.1038/ismej.2015.97

Nobu, M. K., Narihiro, T., Rinke, C., Kamagata, Y., Tringe, S. G., Woyke, T., et al. (2015). Microbial dark matter ecogenomics reveals complex synergistic networks in a methanogenic bioreactor. ISME J. 9, 1710-1722. doi: 10.1038 /ismej.2014.256

Parks, D. H., Imelfort, M., Skennerton, C. T., Hugenholtz, P., and Tyson, G. W. (2015). CheckM: assessing the quality of microbial genomes recovered from isolates, single cells, and metagenomes. Genome Res. 25, 1043-1055. doi: 10.1101/gr.186072.114

Patel, R. K., and Jain, M. (2012). NGS QC Toolkit: a toolkit for quality control of next generation sequencing data. PLoS ONE 7:e30619. doi: 10.1371/journal.pone.0030619

Patil, K. R., Roune, L., and McHardy, A. C. (2012). The PhyloPythiaS web server for taxonomic assignment of metagenome sequences. PLoS ONE 7:e38581. doi: 10.1371/journal.pone.0038581

Pelletier, E., Kreimeyer, A., Bocs, S., Rouy, Z., Gyapay, G., Chouari, R., et al. (2008). Candidatus Cloacamonas acidaminovorans: genome sequence reconstruction provides a first glimpse of a new bacterial division. J. Bacteriol. 190, 2572-2579. doi: 10.1128/JB.01248-07

Rinke, C., Schwientek, P., Sczyrba, A., Ivanova, N. N., Anderson, I. J., Cheng, J. F., et al. (2013). Insights into the phylogeny and coding potential of microbial dark matter. Nature 499, 431-437. doi: 10.1038/nature12352

Salta, M., Wharton, J. A., Blache, Y., Stokes, K. R., and Briand, J. F. (2013). Marine biofilms on artificial surfaces: structure and dynamics. Environ. Microbiol. 15, 2879-2893. doi: 10.1111/1462-2920.12186

Selengut, J. D., and Haft, D. H. (2010). Unexpected abundance of coenzyme F-420dependent enzymes in Mycobacterium tuberculosis and other Actinobacteria J. Bacteriol. 192, 5788-5798. doi: 10.1128/JB.00425-10

Tamura, K., Stecher, G., Peterson, D., Filipski, A., and Kumar, S. (2013). MEGA6: molecular evolutionary genetics analysis version 6.0. Mol. Biol. Evol. 30, 2725-2729. doi: 10.1093/molbev/mst197

Tian, R. M., Wang, Y., Baugouffa, S., Gao, Z. M., Cai, L., Bajic, V., et al. (2014) Genomic analysis reveals versatile heterotrophic capacity of a potentially symbiotic sulfur oxidizing bacterium in sponge. Environ. Microbiol. 16, 3548-3561. doi: 10.1111/1462-2920.12586

van der Wielen, P. W., Bolhuis, H., Borin, S., Daffonchio, D., Corselli, C., Giuliano, L., et al. (2005). The enigma of prokaryotic life in deep hypersaline anoxic basins. Science 307, 121-123. doi: 10.1126/science.1103569

Wang, Q., Garrity, G. M., Tiedje, J. M., and Cole, J. R., (2007). Naive Bayesian classifier for rapid assignment of rRNA sequences into the new bacterial taxonomy. Appl. Environ. Microbiol. 73, 5261-5267. doi: 10.1128/AEM.000 62-07

Wang, Y., Cao, H., Zhang, G., Bougouffa, S., Lee, O. O., Al-Suwailem, A., et al. (2013). Autotrophic microbe metagenomes and metabolic pathways differentiate adjacent Red Sea brine pools. Sci. Rep. 3:1748. doi: 10.1038/srep01748

Werner, J., Ferrer, M., Michel, G., Mann, A. J., Huang, S., Juarez, S., et al. (2014). Halorhabdus tiamatea: proteogenomics and glycosidase activity measurements identify the first cultivated euryarchaeon from a deep-sea anoxic brine lake as potential polysaccharide degrader. Environ. Microbiol. 16, 2525-2537. doi: $10.1111 / 1462-2920.12393$

Zhang, W., Wang, Y., Bougouffa, S., Tian, R., Cao, H., Li, Y., et al. (2015). Synchronized dynamics of bacterial niche-specific functions during biofilm development in a cold seep brine pool. Environ. Microbiol. 17, 4089-4104. doi: $10.1111 / 1462-2920.12978$

Zhang, W., Wang, Y., Lee, O. O., Tian, R., Cao, H., Gao, Z., et al. (2013). Adaptation of intertidal biofilm communities is driven by metal ion and oxidative stresses. Sci. Rep. 3:3180. doi: 10.1038/srep03180

Zhang, W. P., Wang, Y., Tian, R. M., Bougouffa, S., Yang, B., Cao, H. L., et al. (2014). Species sorting during biofilm assembly by artificial substrates deployed in a cold seep system. Sci. Rep. 4:6647. doi: 10.1038/srep 06647

Conflict of Interest Statement: The authors declare that the research was conducted in the absence of any commercial or financial relationships that could be construed as a potential conflict of interest.

Copyright (c) 2016 Zhang, Ding, Yang, Tian, Gu, Luo and Qian. This is an openaccess article distributed under the terms of the Creative Commons Attribution License (CC BY). The use, distribution or reproduction in other forums is permitted, provided the original author(s) or licensor are credited and that the original publication in this journal is cited, in accordance with accepted academic practice. No use, distribution or reproduction is permitted which does not comply with these terms. 